\title{
Canine Diffuse Lepidic Predominant Adenocarcinoma
}

\author{
Cristina Krauspenhar Rossato ${ }^{1}$, Letícia Fiss', Ana Cristina Sotili', \\ Evelyn de Oliveira ${ }^{2}$, Fabrício Camargo ${ }^{2}$ \& Danieli Brolo Martins ${ }^{2}$
}

\begin{abstract}
Background: Due to their abundant vascular supply, the lungs are a usual metastatic site, with primary lung cancer presenting a low prevalence in dogs and cats. Among the primary pulmonary tumors afflicting dogs, lepidic carcinoma is the most common and can be classified by site of onset. Lepidic predominant adenocarcinoma is characterized by the proliferation of neoplastic cells along the pulmonary alveoli and pre-existing alveolar structures. Few reports of this specific tumor were found in the literature, so the objective of this study is to describe the clinical, pathological, and immunohistochemical findings of a dog with predominantly diffuse lepidic adenocarcinoma.

Case: A 14-year-old, female, spayed, mixed-breed canine with a history of progressive weight loss, cough, respiratory distress, and pain while eating was brought in for a consultation. A presumptive diagnosis of pneumonia was suggested. However, in spite of treatment, the dog died, and a necropsy was performed. Upon gross examination, the lungs were diffusely enlarged, whitish, and stiffened with large and highly vascular nodules. Histopathological findings showed proliferation of neoplastic cells along the vascular walls and within the bronchioalveolar structures, with moderate fibrovascular stroma. The growth patterns resembled the pre-existing alveolar structures, with papillary protrusions into the alveolar lumen. After immunohistochemical evaluation, the definitive diagnosis was diffuse lepidic predominant adenocarcinoma. This tumor is uncommon and difficult to diagnose in the clinical veterinary routine, so it should be considered in the differential diagnosis of respiratory conditions in canines, especially in elderly animals with chronic clinical signs not responsive to antibiotic therapy. Complementary diagnostic examinations such as imaging, laboratory tests, and biopsy are indispensable to the early diagnosis of this lung neoplasm.
\end{abstract}

Discussion: Due to its insidious character, primary lung cancer is underdiagnosed in veterinary medicine and is regularly an incidental x-ray finding. Affected dogs have no clinical signs, and this poses a diagnostic challenge. Primary lung cancer tends to be more prevalent in older and purebred animals. The most frequent clinical sign in dogs is a chronic nonresponsive cough, which can be misdiagnosed as another respiratory disease. Hematological findings, such as anemia, neutrophilia, and lymphopenia, can be related to tumor progression. Most reported cases of lepidic adenocarcinoma in dogs have a single pulmonary nodule, and the diffuse form is less common. In this case report, besides the presence of a single pulmonary nodule, there was also widespread involvement of the parenchyma. Histopathology was compatible with lepidic predominant adenocarcinoma, and immunohistochemistry was positive for pan-cytokeratin, confirming the epithelial origin of the tumor, and negative for TFF-1. Despite being a highly specific marker for lung cancer in dogs, TFF-1 has variable sensitivity among the different types of lung cancer. Fixation time and the location of the tumor sections may have contributed to the seronegative result obtained. In conclusion, lepidic adenocarcinoma is a primary lung neoplasia that is uncommon and presents a diagnostic challenge to veterinary clinicians. Therefore, it should be included as a differential diagnosis in older, purebred animals presenting chronic respiratory clinical signs that are refractory to treatment.

Keywords: primary lung cancer, histopathology, immunohistochemical. 


\section{INTRODUCTION}

Primary lung tumors are uncommon tumors in domestic animals, and its prevalence among dogs and cats is about $0.1-0.9 \%$ [3]. It could be divided by its origin as epithelial or mesenchymal and classified according to the site of onset as bronchogenic, bronchiolar, alveolar, or bronchial gland [11]. Among these, adenocarcinoma presented an incidence of 13-15\% [10]. Usually, it has a peripheric presentation, developing near the pleura in the form of multiple masses or single nodules, and its lepidic pattern is characterized by the proliferation of neoplastic cells along the pulmonary alveoli and pre-existing bronchioloalveolar structures [11]. Its diffuse form is rare [6].

Older dogs with an average age of 10 years are the most affected, with a higher incidence in larger breeds such as Labrador Retriever, Bernese Mountain Dog, Boxer and Doberman Pinscher [7]. Approximately 25\% of the animals present no clinical signs, and the tumor is an incidental finding. However, dry cough, exercise intolerance, and dyspnea [7] are the most common clinical signs.

Changes in the hematologic or biochemical profile are nonspecific. Pleural effusion is not a common finding, and in such cases, the analysis of the fluid obtained by thoracentesis is recommended. Normally, the fluid varies from serous to hemorrhagic [9]; although it is worth remembering that the definitive diagnosis is only obtained through histopathological analysis [11].

Few reports of this specific tumor were found in the veterinary literature. Thus, this work describes the clinical, pathological, and immunohistochemical findings of a canine with predominantly diffuse lepidic adenocarcinoma.

\section{CASE}

A 14-year-old, female, spayed, mixed-breed (MB) canine, weighing $6.5 \mathrm{~kg}$, with a history of progressive weight loss, cough, respiratory distress, and pain while eating was brought in for consultation. The owner reported that due an earlier diagnosis of pneumonia, antibiotic therapy was instituted without apparent improvement. The dog also had presented hyporexia and hypodipsia. Upon anamnesis, intense dyspnea was noticed.

Imaging and laboratory testing were conducted. Radiography showed extensive radiopaque areas in the right lung. Hemogram pointed to slight anemia, while the leukogram showed a mild neutrophilia followed by lymphopenia.
The presumptive diagnosis was pneumonia and the patient were hospitalized and treatment was instituted. After seven days of hospitalization, the dog died, and after authorization from the owner, a necropsy was performed.

Macroscopically, the lungs were diffusely enlarged, whitish, and firm. There was a large, highly vascular nodule, $4 \times 3 \times 3 \mathrm{~cm}$ in size, located at the right cranial lobe, with central depression associated with an area of cystic necrosis (Figure 1A). On gross examination, a homogeneous, diffuse, firm, and whitish mass, with a hepatized appearance, was seen (Figure 1B). The other organs did not present notable macroscopic alterations. Histological findings included a proliferation of neoplastic cells along the alveolar walls and within the bronchioalveolar structures, with moderate fibrovascular stroma. This growth pattern resembled the pre-existing alveolar structure (Figure 1C); eventually, these cells protruded into the alveolar lumen, assuming papillary forms (Figure 1D). Neoplastic cells were mostly of cuboid or low columnar shape, but in areas with moderate pleomorphism and extensive areas of invasiveness, they varied in size and shape, with abundant eosinophilic cytoplasm, large nuclei, ranging from round to ovoid. Mitoses were infrequent.

Immunohistochemical evaluation was performed by the streptavidin-biotin-peroxidase (SBPT) technique in centrally located sections of the pulmonary parenchyma. Neoplastic cells demonstrated strongly positive and uniform cytoplasmic marking for pan-cytokeratin (Figure 1E). Sections tested for anti-thyroid transcription factor 1 (TFF-1) were negative.

The definitive diagnosis of diffuse lepidic predominant adenocarcinoma, in this case, was based on the histopathological and immunohistochemical findings on the material obtained through necropsy, in conjunction with imaging and laboratory tests.

\section{DISCUSSION}

Due to its insidious character, primary lung cancer is underdiagnosed, in veterinary medicine. One in four dogs affected by this disease have no clinical signs, which makes diagnosis a challenge. Usually, it is an incidental radiography finding that can be mistaken by other diseases that affect the lungs, such as pneumonia [4].

Although rare in animals, including dogs, primary lung tumors occur more frequently in older animals, at 10 years of age on average. Mixed-breed 


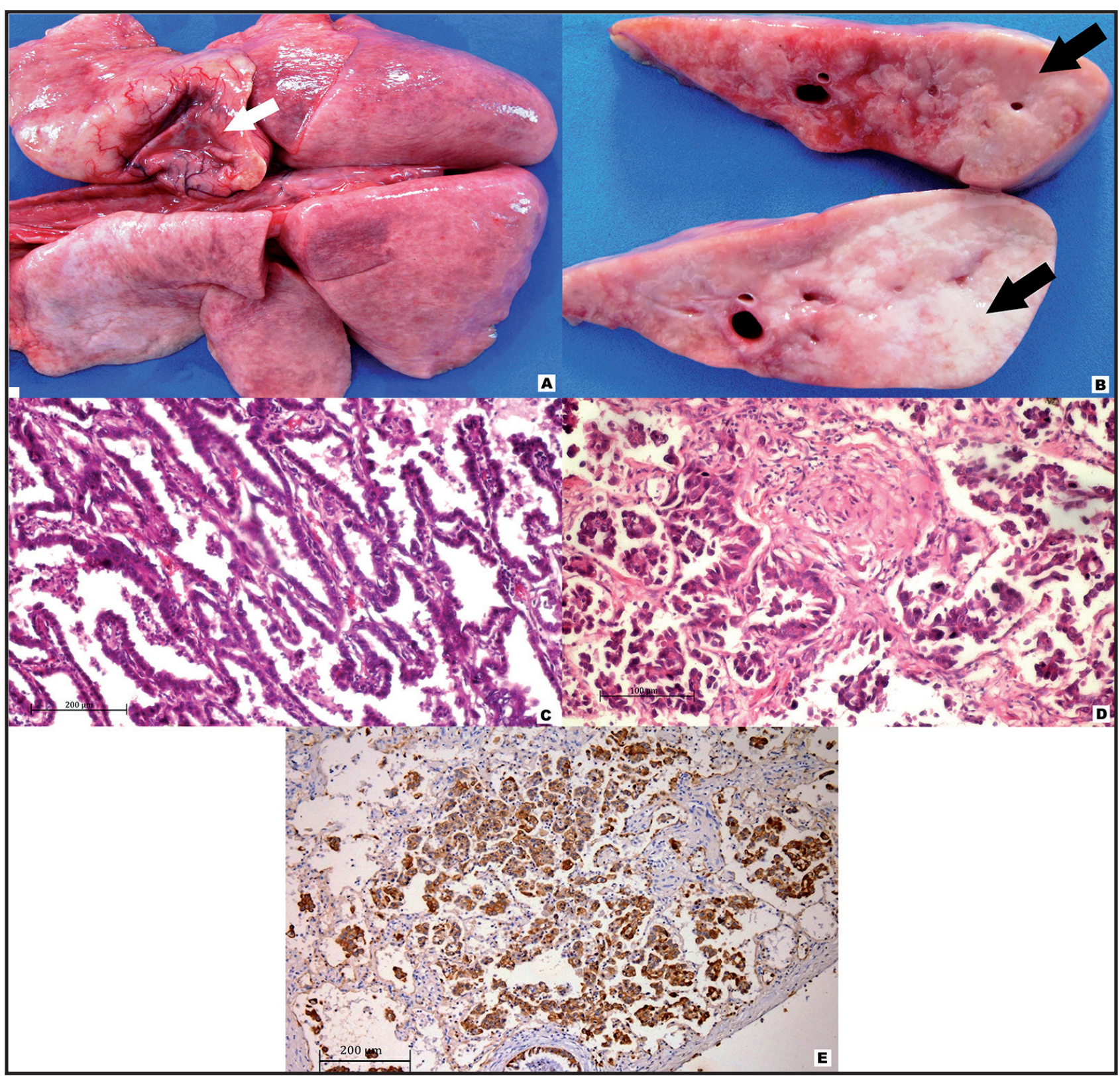

Figure 1. Canine lepidic predominant adenocarcinoma. A- Diffuse whitish homogenous mass, with large nodule presenting a central depression (white arrow). B- On gross examination, hepatization can be seen (parenchyma stiffening - black arrows). C- Histologically, neoplastic cells are arranged in alveoli, resembling the alveolar pre-existing structure [H\&E; 10x]. D- Eventually, these cells protrude into the alveolar lumen [H\&E; 20x]. E- Strongly marking for pancytokeratin on neoplastic cells cytoplasm. Immunohistochemical, streptavidin-biotin-peroxidase (SBPT) method [Harris haematoxylin counterstaining; 10x].

dogs are less predisposed [9], which makes this case more unique and relevant.

The most frequent clinical sign in dogs with this disease is chronic cough, non-responsive to antibiotics, reinforcing the silent behavior of the disease $[6,7]$. This fact impairs an early intervention since it can be confused with other respiratory diseases, thereby delaying appropriate care for the patient.

The hematological findings observed in this case may be secondary to the development of lung neoplasia. Anemia is a recurrent finding in cancer patients and may be associated with pathogenic mechanisms that predispose to bleeding, destruction, or deficit in the production of erythrocytes by the bone marrow [1]. As for the leukogram, neutrophilia with consequent lymphopenia may be due to both pain stress and respiratory distress, as well as a systemic response to the inflammatory cytokines secreted by the tumor [5]. Most of the reported cases of lepidic predominant adenocarcinoma in dogs have a single peripheral pulmonary nodule $[6,11]$. The diffuse form is less common and compromises a large part of the pulmonary 
parenchyma, and could be mistaken by pneumonia in the clinical and radiographic evaluation [2,6]. In the reported case, despite the presence of a single nodule in the right cranial lobe of the lung, there was also a generalized involvement of the parenchyma, which made clinical diagnosis difficult.

Histopathological evaluation was compatible with lepidic predominant adenocarcinoma, in accordance with other studies that demonstrated this neoplasia is characterized by the proliferation of neoplastic cells along the pulmonary alveoli and pre-existing bronchioloalveolar junctions [3,6,11]. This classification of lung tumors in humans and animals is based on the site of origin and/or histological patterns [11]. In this case, the initial evaluation using the immunohistochemistry technique demonstrated positive immunostaining for pan-cytokeratin, confirming the epithelial origin of the tumor. Nevertheless, in order to prove the primary origin of the lung neoplasia, an analysis for TTF-1 was performed, obtaining a negative result. Although it is a highly specific marker for the diagnosis of primary lung cancer in dogs, the sensitivity for the positive cell immunostaining is variable amongst the various neoplastic types found in this tissue. One study demonstrated the positive immunoreactivity of TFF1 to canine lung adenocarcinoma was approximately $77.4 \%$ [8]. This may be correlated with the prolonged fixation time of the material [8] and with the location of the analyzed tumor sections $[3,6,8]$.
Neoplastic cells located at the periphery of lung tumor masses color more easily and strongly than those of central regions $[6,8]$. The tumor sections assessed for TTF-1 immunostaining were collected from the center of the mass, which may justify the absence of marking.

The lepidic predominant adenocarcinoma is a primary lung cancer that is uncommon and presents a difficult diagnosis in veterinary clinical routine. Therefore, it should be considered in the differential investigation of respiratory diseases in canines, especially in elderly, purebred animals presenting chronic clinical signs that do not respond to antibiotic therapy. Additionally, the importance of complementary diagnostic tests, such as imaging, laboratory, and biopsy, should be considered, and the histopathological analysis is indispensable for early diagnosis of this lung cancer. Immunohistochemical evaluation is important for an assessment of tumor origin. TTF-1 is the marker of choice in these cases. However, it should be emphasized that this antibody can demonstrate negative marking correlated to the evaluation site of the harvested material.

Acknowledgments. We want to acknowledge the pathologists of the Department of Veterinary Pathology of the Federal University of Rio Grande do Sul (UFRGS), Luciana Sonne and David Driemeier, for the performing of the IHC technique in this case report.

Declaration of interest. The authors report no conflicts of interest. The authors alone are responsible for the content and writing of the paper.

\section{REFERENCES}

1 Bergman P.J. 2013. Paraneoplastic Syndromes. In: Withrow S.J., Vail D.M. \& Page R.L. (Eds). Withrow MacEwen's Small Animal Clinical Oncology. St. Louis: Elsevier Saunders, pp.83-97.

2 Bertazzolo W., Zuliani D., Pogliani E., Caniatti M. \& C. Bussadori C. 2002. Diffuse bronchiolo-alveolar carcinoma in a dog. Journal of Small Animal Practice. 43(6): 265-268.

3 Caswell J. \& Williams K. 2016. Respiratory system. In: Maxie M.G. (Ed). Jubb, Kennedy Palmer's Pathology of Domestic Animals. v2. 6th edn. St. Louis: Elsevier, pp.465-591.

4 Conti M.B., Marchesi M.C., Angeli G., Lepri E., Marinetti C. \& Rueca F. 2010. A case of primary papillary disseminated adenocarcinoma of canine lung. Veterinary Research Communications. 34(S1): 111-115.

5 Harvey J.W. 2012. Evaluation of Leukocytic Disorders. In: Harvey J.W. (Ed). Veteterinary Hematology. St. Louis: Elsevier, pp.122-176.

6 Lucena R.B., Maia L.A., Dantas A.F.M., Nobre V.M.T., Macêdo J.T.S.A., Galiza Filho E.M. \& Barros C.S.L. 2011. Carcinoma bronquíolo-alveolar difuso em um cão: aspectos clínico-patológicos e imuno-histoquímicos. Ciência Rural. 41(1): 160-163.

7 Ogilvie G.K., Haschek W.M., Withrow S.J., Richardson R.C., Harvey H.J., Henderson R.A., Fowler J.D., Norris A.M., Tomlinson J. \& McCaw D. 1989. Classification of primary lung tumors in dogs: 210 cases (1975-1985). Journal of the American Veterinary Medical Association. 195(1): 106-108. 
8 Ramos-Vara J.A., Miller M.A. \& Johnson G.C. 2005. Usefulness of Thyroid Transcription Factor-1 Immunohistochemical Staining in the Differential Diagnosis of Primary Pulmonary Tumors of Dogs. Veterinary Pathology. 42(3): 315-320.

9 Rebhun R.B. \& Culp W.T.N. 2013. Pulmonary Neoplasia. In: Withrow S.J., Vail D.M. \& Page R.L. (Eds). Withrow MacEwen's Small Animal Clinical Oncology. St. Louis: Elsevier Saunders, pp.453-462.

10 Wilson D.W. 2017. Tumors of the Respiratory Tract. In: D.J. Meuten (Ed). Tumors in Domestic Animals. 5th edn. Raleigh: Wiley Blackwell, pp.467-498.

11 Wilson D.W. \& Dungworth D.L. 2002. Tumors of the respiratory tract. In: D.J. Meuten (Ed). Tumors in Domestic Animals. 4th edn. Ames: Iowa State Press, pp.365-400. 